\title{
Disease-related pregnancy concerns and reproductive planning in women with inflammatory bowel diseases
}

\author{
Lori M Gawron, ${ }^{1}$ Adina R Goldberger, ${ }^{2}$ Andrew J Gawron, ${ }^{3}$ \\ Cassing Hammond, ${ }^{4}$ Laurie Keefer ${ }^{5}$
}

\begin{abstract}
${ }^{1}$ Assistant Professor, Department of Obstetrics and Gynecology, University of Utah, Salt Lake City, UT, USA

${ }^{2}$ Medical Student, Northwestern University Feinberg School of Medicine, Chicago, IL, USA ${ }^{3}$ Assistant Professor, Division of Gastroenterology, University of Utah, Salt Lake City, UT, USA

${ }^{4}$ Associate Professor, Department of Obstetrics and Gynecology, Northwestern University Feinberg School of Medicine, Chicago, IL, USA ${ }^{5}$ Associate Professor, Division of Gastroenterology and Hepatology, Northwestern University Feinberg School of Medicine, Chicago, IL, USA
\end{abstract}

\section{Correspondence to}

Dr Lori M Gawron, Department of Obstetrics and Gynecology, University of Utah, 30n 1900e Room 2B-200, Salt Lake City, UT 84132, USA;

lori.gawron@hsc.utah.edu

Received 12 June 2014 Revised 18 March 2015 Accepted 19 March 2015 Published Online First 22 April 2015
CrossMark

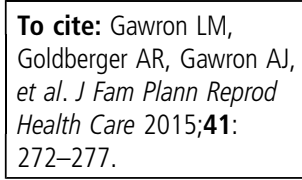

\begin{abstract}
Background Women with inflammatory bowel diseases (IBD) endorse disease-related pregnancy concerns that influence parity. Improvements in IBD management have potentially altered reproductive planning. Additionally, the proportion of American women who choose not to have children is increasing.
\end{abstract}

Aim To explore the effect of disease-related pregnancy concerns on parity and reproductive planning in a subset of women with IBD.

Design and setting Cross-sectional qualitative phone survey in an academic gastroenterology practice.

Methods Questions included demographics, medical and reproductive history, future pregnancy plans, and if IBD affected pregnancy decision-making. Qualitative data were coded and frequencies and proportions calculated.

Results The 129 female participants (31\% response rate) were predominately white $(85 \%)$, had at least some college education (97\%) and a mean age of 34.3 years [standard deviation 6.2]. Some $60 \%$ had Crohn's disease and 30\% had undergone IBD-related surgery. Half were nulliparae, 53\% reported IBD-related pregnancy concerns and $57 \%$ desired future pregnancy. Women who desired a future pregnancy and had IBD-related concerns had higher parity than those without concerns ( $p=0.02$ ). Women desiring a future pregnancy and those with Crohn's disease had increased IBD-related concerns. Only four (3.1\%) women identified IBD-related concerns that led to a smaller family size than desired.

Conclusions IBD-related concerns appear to be less likely to affect a woman's planned family size than previously reported. Concern about adverse pregnancy outcomes is more common in women with Crohn's disease and those desiring future pregnancy, suggesting a need for targeted counselling to moderate risk perception.
Key message points

- Disease-related pregnancy concerns of women with inflammatory bowel diseases (IBD) are less likely to affect planned family size than previously reported.

- Women with Crohn's disease are more likely to have IBD-related pregnancy concerns than those with ulcerative colitis, although treatment differences may account for this finding.

- The concern for adverse pregnancy outcomes is more common in women with IBD desiring future pregnancy, suggesting a need for targeted counselling to moderate risk perception.

\section{INTRODUCTION}

Inflammatory bowel diseases (IBD) are chronic, relapsing autoimmune disorders primarily comprised of Crohn's disease and ulcerative colitis. Typical IBD symptoms include abdominal pain, bloating, diarrhoea, faecal urgency, hematochezia, and malaise or fatigue. Severe disease can lead to colonic perforation, bowel obstruction due to adhesive disease, strictures and fistulae necessitating surgical interventions. Each patient has a different anatomical location of disease, severity of symptoms, and frequency of disease relapse. As no cure for IBD exists, chronic immunosuppression or immunomodulation is used to heal tissue and increase remission time, although quality of life may be altered regardless of disease activity. ${ }^{1}$ There are approximately 1.4 million Americans afflicted by the diseases and 70000 new cases annually; the incidence and prevalence of the 
diseases are increasing worldwide. ${ }^{2}$ IBD is frequently diagnosed in the second to fourth decades, with the highest incidence between the ages of 20 and 29 years - prime reproductive years for women. $^{2}$

Reproductive planning is an important consideration for women with IBD. Adverse pregnancy outcomes, such as preterm delivery, low birth weight, and increased risk of miscarriage, are all associated with the level of disease activity at the time of conception. ${ }^{3-7}$ Women with IBD, who plan their pregnancies in a state of remission, are less likely to experience disease-flares during pregnancy. ${ }^{8}$ The infertility rate for women with IBD who are medically-managed is similar to the general population. The only exception is that women with ulcerative colitis who undergo an ileal-pouch anal anastomosis have an increased rate of infertility post-procedure. ${ }^{9} 10$ The majority of women with well-controlled IBD can be assured of similar pregnancy outcomes to women without the disease.

Despite these data, previous research identified lower parity and higher rates of voluntary childlessness in women with IBD compared to the general population. ${ }^{11-13}$ A USA-based study by Marri et al. ${ }^{11}$ in 2007 identified a higher rate of voluntary childlessness than the general population; a finding potentially confounded by the educational attainment and racial background of the respondents. Some study participants expressed concerns about their disease management in pregnancy, heritability of the disease, or the ability to care for a child in the setting of their medical needs, as reasons for voluntary childlessness.

An Australian group in 2009 also found a higher rate of voluntary childlessness in a study cohort and identified participant disease-related concerns that were out of proportion to actual risk as the potential cause. These concerns included fear of congenital anomalies or disease heritability, medication teratogenicity, or physician advice against pregnancy due to IBD. ${ }^{12}$ A recent Spanish study found personal choice was the most common reason for voluntary childlessness in participants with IBD, with only $8 \%$ citing disease-related concerns as a cause for their decision. ${ }^{13}$ Overall, these studies relied upon mail-in survey responses for small cohorts, and many participants had yet to begin or complete their intended family size; therefore a direct comparison to population-based fertility statistics is flawed.

Over the past decade, medical management of IBD has changed with the addition of biological therapies and increasing pregnancy safety data. ${ }^{14}$ The change in management of this chronic disease has potentially altered IBD-related pregnancy concerns for women, allowing them the hope of attaining disease remission prior to childbearing. In addition, the average age at first childbirth has increased in the USA, as well as the proportion of women who choose not to have children or have a smaller family size. Currently, 1 in 5 women will complete their reproductive years childless, compared to 1 in 10 women in the 1970s. ${ }^{15} 16$ Recent research identified associations between childlessness and poor mental and physical quality of life in later years; findings that may have additional implications for women with chronic medical comorbidities. ${ }^{17}{ }^{18}$ As the only study in the USA was completed early in the era of biological therapy, ${ }^{11}$ and prior studies primarily assessed current parity, not planned family size, we sought to explore disease-related pregnancy concerns and their effect on parity and future reproductive planning in a subset of women with IBD.

\section{METHODS}

The Institutional Review Board of Northwestern University approved this study. An enquiry to Northwestern University Enterprise Data Warehouse identified all women, aged 18-45 years, with Crohn's disease or ulcerative colitis, who accessed care with the Northwestern Medical Faculty Foundation's academic gastroenterology practice from 2010 to 2012. A manual chart review confirmed diagnosis and extracted contact information. We mailed potential participants an opt-out letter and then contacted the women by telephone 30 days following the mailing. All potential participants received up to three attempts at telephone contact between March and November 2013 and verbal consent was obtained for survey participation.

Telephone survey questions included demographics, medical and reproductive history, details regarding disease activity, and plans for future pregnancy. Open-ended qualitative questions probed if and how disease-related pregnancy concerns had affected reproductive decision-making in the past or for future pregnancies. We documented qualitative responses on disease-related pregnancy concerns verbatim and coded responses numerically. The data were hand written on data entry sheets by the interviewer, manually imported into Microsoft Excel ${ }^{\mathrm{TM}}$ spreadsheets, and a separate team member checked the imported data for accuracy in 10 randomly selected subjects. Data were analysed with Stata V.11 ${ }^{\mathrm{TM}}$ (StataCorp, College Station, TX, USA).

All quantitative and descriptive statistics were calculated and frequencies and proportions reported. For continuous data, normalcy was assessed and mean or median reported, as appropriate. Comparisons were made by disease via $t$-test for means, Fisher's exact test or Chi-square $\left(\chi^{2}\right)$ to assess distributions between groups based upon group numbers, Wilcoxon rank sum test for medians, or two-sample test of proportions (Table 1). Median parity for those who desired a future pregnancy was compared with Wilcoxon rank sum test to those not desiring pregnancy by whether reproductive concerns affected that decision. In order to evaluate this difference further, parity was stratified by number of pregnancies $(0,1$ or 2 or more) and 
Table 1 Participant sociodemographic, disease, and reproductive characteristics by diagnosis

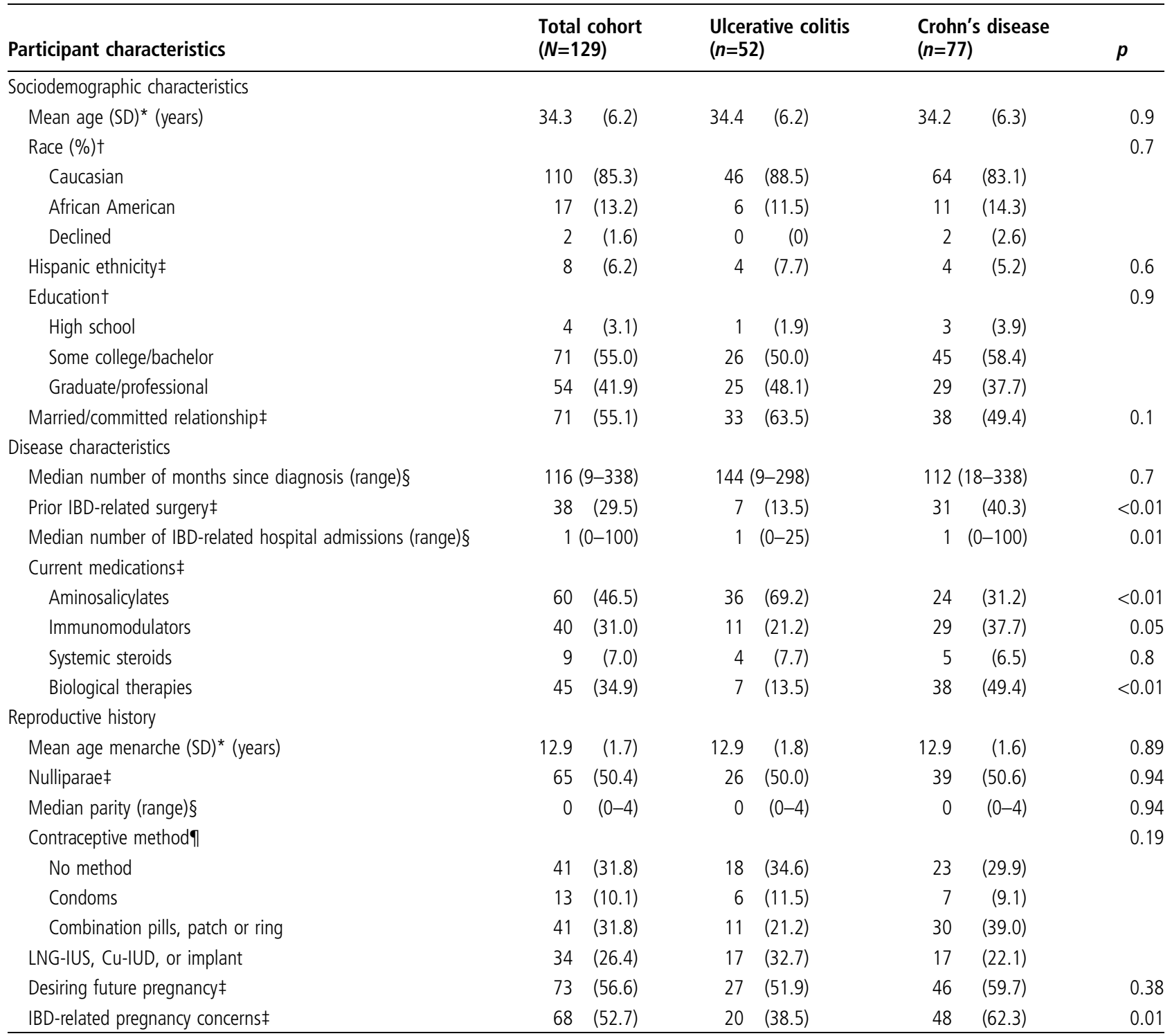

Entries in the table represent the number of participants (percentage) unless otherwise specified.

IBD medication classes: Aminosalicylates include balsalazide, sulfasalazine, mesalamine and olsalazine. Immunomodulators include azathioprine,

6-mercaptopurine and methotrexate. Topical or systemic steroids include hydrocortisone enemas, suppositories and other formulations, budesonide, prednisone, methylprednisolone and prednisolone. Biological therapies include infliximab, adalimumab, certolizumab and natalizumab.

${ }^{*} t$-test.

†Fisher's exact test.

$\ddagger$ Two-sample test of proportions.

$\S$ Wilcoxon rank sum test.

IChi-square $\left(\chi^{2}\right)$ test.

Cu-IUD, copper intrauterine device; IBD, inflammatory bowel diseases; LNG-IUS, levonorgestrel intrauterine system; SD, standard deviation.

Fisher's exact test used to assess for differences between pregnancy desire and the impact of IBD-related pregnancy concerns (Table 2).

\section{RESULTS}

A total of 420 opt-out letters were mailed and not returned to sender, 50 telephone numbers were wrong or disconnected, 80 women verbally declined participation, and 161 women had three telephone attempts without contact. A total of 129 participants (31\% response rate) completed the telephone survey.
The participants were predominately white (85\%), with at least some college education (97\%), and a mean age of 34.3 years [standard deviation (SD) 6.2 years, range $19-45$ years]. Some $60 \%$ had Crohn's disease and 40\% ulcerative colitis. Crohn's disease participants were more likely to have been hospitalised for their disease $(p=0.01)$, undergone IBD-related surgery $(p<0.01)$, or be on biological therapy $(p<0.01)$ than ulcerative colitis participants. Ulcerative colitis participants were more likely to use aminosalicylates for medical management of their 
disease $(p<0.01)$. Half the participants had at least one child and $57 \%$ desired a future pregnancy. Disease type $(p=0.19)$, concerns regarding IBD and pregnancy $(p=0.43)$, and the desire for a future pregnancy $(p=0.11)$ did not significantly influence participants to choose a more effective method of contraception: $31.8 \%$ of participants used no method. Some $53 \%$ of participants cited disease-related concerns that affected decision-making regarding pregnancy. Participants with Crohn's disease were significantly more likely to report disease-related concerns $(62 \%)$ than those with ulcerative colitis $(38 \%)$ $(p=0.01)$ (Table 1).

Of the 73 women who desired a future pregnancy, the median parity was higher in those with IBD-related concerns (0, range 0-3) compared to those without concerns (0, range $0-2) \quad(p=0.02)$. Although reaching statistical significance, there is no clinically significant difference in this finding. Of women who had completed their family, the final parity was not significantly different between those who had IBD-related pregnancy concerns compared to those who did not $(p=0.8)$. Although not statistically significant, stratifying by parity categories identified a higher proportion of those with a future pregnancy desire with IBD-related concerns to be nulliparas compared to those without concerns (Table 2).

Overall, women desiring a future pregnancy were more concerned about adverse pregnancy outcomes $(69.1 \%)$ than those who did not $(30.9 \%)$. The concerns most frequently reported were on the effect of medications in pregnancy, the effect of pregnancy on disease activity, heritability of the disease, and the potential of infertility. Only four (3\%) women identified IBD-related concerns that led to a smaller family size than desired. As women desiring a future pregnancy were more likely to report concerns, we compared the reported concerns by disease type.
The sample sizes were small for comparisons, yet only the concern regarding disease heritability was significantly different between Crohn's disease and ulcerative colitis participants who desired a future pregnancy (Figure 1).

\section{DISCUSSION}

This study explored the effect of disease-related pregnancy concerns on both planned and completed family size in a subset of women with IBD. Reproductive planning is multifaceted for every woman, ${ }^{19}$ and these data suggest IBD-related concerns are less likely to be isolated factors that affect a woman's planned family size than previously reported. ${ }^{11}{ }^{12}$ The median parity for those who had completed their family and reported disease-related concerns was no different from those who had completed their family without concerns. The concern for adverse pregnancy outcomes is more common in women with Crohn's disease compared to ulcerative colitis and in those desiring future pregnancy, suggesting a need for targeted counselling to moderate risk perception.

The concerns reported by the women in this study are consistent with previous studies, ${ }^{11-13}$ yet do not appear to interfere with reproductive planning. The median parity for women who have completed their family in this study is not outside of the norm for an average family size in the USA or other developed nations at this time,${ }^{20}$ although population-based data for women with IBD would be necessary to make a meaningful comparison. Women are realistic in considering the impact of their disease on their ability to become pregnant, carry the child, deliver a developmentally intact baby, and care for that child in the context of other life responsibilities. These concerns are not unlike any other woman's concerns, with or without a chronic disease, as she considers a future

Table 2 Comparison of participant parity by future pregnancy desire and inflammatory bowel disease-related pregnancy concerns

\begin{tabular}{|c|c|c|c|}
\hline Parity and future pregnancy & $\begin{array}{l}\text { IBD concerns have affected } \\
\text { pregnancy decisions }\end{array}$ & $\begin{array}{l}\text { IBD concerns have not } \\
\text { affected pregnancy decisions }\end{array}$ & $p$ \\
\hline \multicolumn{4}{|l|}{ Desires future pregnancy } \\
\hline Median parity (IQR, range) ${ }^{*}$ & $0(1,0-3)$ & $0(0,0-2)$ & 0.02 \\
\hline Proportions of parity categories $[n(\%)] \dagger$ & & & 0.08 \\
\hline Parity $=0$ & $22 \quad(84.6)$ & $28 \quad(59.6)$ & \\
\hline Parity $=1$ & $3 \quad(11.5)$ & $10 \quad(21.3)$ & \\
\hline Parity $=2+$ & $1 \quad(3.9)$ & $9 \quad(19.2)$ & \\
\hline \multicolumn{4}{|l|}{ No desire for future pregnancy } \\
\hline Median parity (IQR, range) ${ }^{*}$ & $1(3,0-4)$ & $2(2,0-4)$ & 0.75 \\
\hline Proportions of parity categories $[n(\%)] \dagger$ & & & 0.57 \\
\hline Parity $=0$ & $12 \quad(34.3)$ & $10 \quad(47.6)$ & \\
\hline Parity $=1$ & $3 \quad(8.6)$ & $1 \quad(4.8)$ & \\
\hline Parity $=2+$ & $20 \quad(57.1)$ & $10 \quad(47.6)$ & \\
\hline
\end{tabular}

*Wilcoxon rank sum test $t$-test.

†Fisher's exact test.

IBD, inflammatory bowel diseases; IQR, interquartile range. 


$$
\begin{aligned}
& \text { QUlcerative colitis } \quad \square \text { Crohn's disease } \\
& (n=27) \quad(n=46)
\end{aligned}
$$

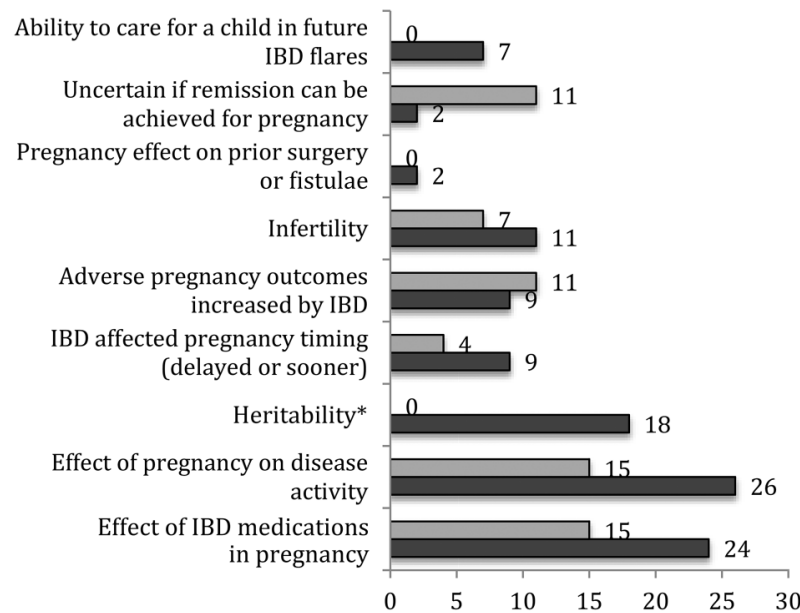

Figure 1 Proportion of participants with a future pregnancy desire reporting inflammatory bowel (IBD)-related pregnancy concerns by IBD diagnosis. ${ }^{*} p<0.05$.

pregnancy. ${ }^{19} 2122$ Rather, the concerns imply an insight and thoughtfulness in the planning of the woman's family. In addition, the increased concerns expressed by the participants with Crohn's disease might reflect previous surgical exposure or the relatively new and limited data on some biological therapies used for this disease. Regardless, the desire to have children appears to outweigh any disease-related concerns in the current era of treatment, at least for this cohort of patients.

The significant difference between the proportions of participants with disease-related concerns who desire a future pregnancy compared to those who do not plan any future children highlights an area for educational interventions. An Australian group showed that targeted education by a gastroenterologist increases the knowledge of study participants with IBD on pregnancy-related topics. ${ }^{23}$ Replicating such patient and provider interventions may assist in moderating disease-related concerns and allow for healthy choices around reproductive planning for individuals affected by IBD. It is unknown whether educational interventions would impact final reproductive planning on a broad scale, but would allow for preconception counselling and informed decision-making.

The limitations in this study are inherent to the cross-sectional cohort study design. The small sample size and selection bias could account for the findings and limit generalisability, as the majority of participants were Caucasian and educated. Disease-specific concerns may have a greater impact on reproductive goals in women with limited socioeconomic means, lack of insurance, or lower education levels. The mode of data collection required English ability and access to a telephone; therefore the results might not be representative of non-English speaking populations or groups without stable telephone access. No comparisons were available between those who participated and those who were not reached for the telephone survey. In addition, the open-ended responses of participants targeted disease-specific concerns, and did not explore socioeconomic or other reproductive planning considerations. No comparative group of healthy women was included, as this study aimed to explore the unique considerations of this special population. The reported data do not assess final reproductive outcomes; only planned family size. In addition, women who stated they did not desire a future pregnancy could also change plans or have an unintended pregnancy.

Despite these limitations, the data support continued efforts to educate women with IBD on issues surrounding reproductive planning. Mitigating perceived risks might allow for women to choose a family size based upon personal desire, rather than fear of adverse disease outcomes. Further research to assess best patient and provider educational interventions, as well as comparative studies across the spectrum of IBD severity, could inform clinical practice guidelines and minimise misperceptions regarding risk of pregnancy for women with IBD.

Funding NIH/NICHD Grant K12 HD050121.

Competing interests None.

Ethics approval Northwestern University IRB.

Provenance and peer review Not commissioned; externally peer reviewed.

\section{REFERENCES}

1 Keefer L, Taft TH, Kiebles JL, et al. Gut-directed hypnotherapy significantly augments clinical remission in quiescent ulcerative colitis. Aliment Pharmacol Ther 2013;38:761-771.

2 Molodecky NA, Soon IS, Rabi DM, et al. Increasing incidence and prevalence of the inflammatory bowel diseases with time, based on systematic review. Gastroenterol 2012;142:46-54.

3 Riis L, Vind I, Politi P, et al. Does pregnancy change the disease course? A study in a European cohort of patients with inflammatory bowel disease. Am J Gastroenterol 2006;101: 1539-1545.

4 Fonager K, Sorensen HT, Olsen J, et al. Pregnancy outcome for women with Crohn's disease: a follow-up study based on linkage between national registries. Am J Gastroenterol 1998;93:2426-2430.

5 Norgard BM. Birth outcome in women with ulcerative colitis and Crohn's disease, and pharmacoepidemiological aspects of anti-inflammatory drug therapy. Dan Med Bull 2011;58:B4360.

6 Stephansson O, Larsson H, Pedersen L, et al. Crohn's disease is a risk factor for preterm birth. Clin Gastroenterol Hepatol 2010;8:509-515.

7 Getahun D, Fassett MJ, Longstreth GF, et al. Association between maternal inflammatory bowel disease and adverse perinatal outcomes. J Perinatol 2014;34:435-440.

8 Heetun ZS, Byrnes C, Neary P, et al. Review article: reproduction in the patient with inflammatory bowel disease. Aliment Pharmacol Ther 2007;26:513-533. 
9 Tavernier N, Fumery M, Peyrin-Biroulet L, et al. Systematic review: fertility in non-surgically treated inflammatory bowel disease. Aliment Pharmacol Ther 2013;38: 847-853.

10 Rajaratnam SG, Eglinton TW, Hider P, et al. Impact of ileal pouch-anal anastomosis on female fertility: meta-analysis and systematic review. Int J Colorectal Dis 2011;26:1365-1374.

11 Marri SR, Ahn C, Buchman AL. Voluntary childlessness is increased in women with inflammatory bowel disease. Inflamm Bowel Dis 2007;13:591-599.

12 Mountifield R, Bampton P, Prosser R, et al. Fear and fertility in inflammatory bowel disease: a mismatch of perception and reality affects family planning decisions. Inflamm Bowel Dis 2009;15:720-725.

13 Manosa M, Navarro-Llavat M, Marin L, et al. Fecundity, pregnancy outcomes, and breastfeeding in patients with inflammatory bowel disease: a large cohort survey. Scand J Gastroenterol 2013;48:427-432.

14 Yarur A, Kane SV. Update on pregnancy and breastfeeding in the era of biologics. Dig Liver Dis 2013;45:787-794.

15 Pew Research Center. Childlessness Up Among All Women; Down Among Women with Advanced Degrees. 2010. http:// www.pewsocialtrends.org/files/2010/11/758-childless.pdf [accessed 21 April 2014].
16 Hayford SR. Marriage (still) matters: the contribution of demographic change to trends in childlessness in the United States. Demography 2013;50:1641-1661.

17 Graham ML, Hill E, Shelley JM, et al. An examination of the health and wellbeing of childless women: a cross-sectional exploratory study in Victoria, Australia. BMC Womens Health 2011;11:47.

18 Read S, Grundy E, Wolf DA. Fertility history, health, and health changes in later life: a panel study of British women and men born 1923-49. Popul Stud (Camb) 2011;65:201-215.

19 Benzies K, Tough S, Tofflemire K, et al. Factors influencing women's decisions about timing of motherhood. J Obstet Gynecol Neonatal Nurs 2006;35:625-633.

20 The World Factbook. Total fertility rate. 2014. https://www.cia. gov/library/publications/the-world-factbook/fields/2127.html [accessed 15 May 2014].

21 Prunty M, Sharpe L, Butow P, et al. The motherhood choice: themes arising in the decision-making process for women with multiple sclerosis. Mult Scler 2008;14:701-704.

22 Biggs MA, Gould H, Foster DG. Understanding why women seek abortions in the US. BMC Womens Health 2013;13:29.

23 Mountifield R, Andrews JM, Bampton P. It is worth the effort: patient knowledge of reproductive aspects of inflammatory bowel disease improves dramatically after a single group education session. J Crohns Colitis 2014;8:796-801. 